\title{
Application of Neural Networks to Morphological Assessment in Bovine Livestock
}

\author{
Horacio M. González-Velasco, Carlos J. García-Orellana, \\ Miguel Macías-Macías, Ramón Gallardo-Caballero, \\ and Antonio García-Manso \\ CAPI Research Group \\ Politechnic School, University of Extremadura \\ Av. de la Universidad, s/n. 10003 Cáceres - Spain \\ horacio@capi.unex.es
}

\begin{abstract}
In conservation and improvement programs of bovine livestock, an important parameter is morphological assessment, which consist of scoring an animal attending to its morphology, and is always performed by highly-qualified staff.

We present in this paper a system designed to help in morphological assessment, providing a score based on a lateral image of the cow. The system consist of two main parts. First, a feature extractor stage is used to reduce the information of the cow in the image to a set of parameters (describing the shape of the profile of the cow). For this stage, a model of the object is constructed by means of point distribution models (PDM), and later that model is used in the searching process within each image, that is carried out using genetic algorithms (GAs). Second, the parameters obtained are used in the following stage, where a multilayer perceptron is trained in order to provide the desired assessment, using the scores given by experts for selected cows.

The system has been tested with 124 images corresponding to 44 individuals of a special rustic breed, with very promising results, taking into account that the information contained in only one view of the cow is not complete.
\end{abstract}

\section{Introduction}

As for the control and conservation of purity in certain breeds of bovine livestock, European legislation imposes the creation of herd-books, records of the information stored about existing animals of the above-mentioned race. Morphological assessment is one of the parameters that are stored on those herd-books for each pure-bred animal, and will be of great importance for many tasks that are done with them. In general, the morphological assessment consist of scoring an animal considering its external morphology (hence is also known as conformational evaluation or external evaluation) and the similarity of its characteristics to the standard of the race. This score is usually settled between 0 and 100 points, and is conducted by highly-qualified and experienced staff (which are known as 
assessors or, more generally, experts). This personnel must behave in a "neutral way" and they have to be trained in a centraliced manner in order to maintain the criteria.

There are several methods to carry out the morphological assessment, usually considering measurable traits and traits directly evaluated by visual inspection 7. However, with rustic meat-producing breeds, normally breeded in extensive farms, morphological assessment is only based in visual inspection. This method consist of scoring ten traits, each one including several aspects of the standard of the race (see table 1). Those traits are assessed with a continuous value between 1 and 10 points, and the global score is obtained by a weighted sum of the partial scores. The weights are determined by the characteristics of the breed and the objectives to be reached with its control and selection. This is the method applied for the breed considered in this work. A similar method, but in other context, is described in 3 .

Table 1. Traits to be individually assessed for the considered breed. The weights for the final sum are included.

\begin{tabular}{lcc}
\hline Trait to be assessed & Weights (males) & Weights (females) \\
\hline General aspect and figure & 1.6 & 1.6 \\
Development of the body & 1.2 & 1.2 \\
Head & 0.5 & 0.5 \\
Neck, chest, withers and back & 0.5 & 0.4 \\
Thorax and belly & 1.0 & 1.0 \\
Back and loins & 1.5 & 1.3 \\
Hindquarters and tail & 1.2 & 1.3 \\
Thighs and buttocks & 1.3 & 1.3 \\
Extremities & 0.7 & 1.0 \\
Genital organs & 0.5 & - \\
Shape and quality of the udder & - & 0.5 \\
\hline
\end{tabular}

With this method, it is worrisome the great amount of subjectivity involved in morphological assessment. This together with the need of uniformity in criteria, leads us to consider the utility of a semiautomatic system to help morphological assessment, as already discussed in [7. In this work, as well as in the information received from the consulted experts it is suggested that the assessment could be done quite accurately using three pictures corresponding to three positions of the cow (frontal, lateral and rear), and fundamentally analyzing the profiles of the animal in those images. In this paper we present a first approach to an automatic morphological assessment system, based on images of only one of the views: the lateral position.

The proposed method has a classical structure in two stages. First, a feature extraction is performed on the image, with the aim of representing the profile of the cow with a reduced set of parameters. Later, using this parameters, a supervised neural network system is trained with samples of assessments made 
by the experts. This way, their knowledge about assessment can be extracted and their assessments can be approximately reproduced.

For the first stage we use a method based on point distribution models (PDM) [2] and genetic algorithms (GAs) 48, and its aim is to parameterize and extract the contours of the animals in the images. The contours are parameterized by means of PDM, and this parametrization is used, along with a potential image generated from the original colour image, to carry out an efficient search with GAs, as described in [56]. Later, in the second stage, we propose to use the contours extracted for a selected set of cows, and the assessments made by experts for that cows, to train a supervised neural network (a multilayer perceptron [1]). Once the neural network is trained, the complete system can provide an assessment for each image presented at its input.

In section 2 the technique used to search the contours within the images is summarized. Next, section 3 provides details about the neural network used and the training method. The results obtained by applying the whole system to a set of images can be found in section 4, whereas our conclusions and possible improvements or our work are presented in section 5 .

\section{First Stage: Image Analysis}

The contour extraction problem can be converted into an optimization problem considering two steps: the parametrization of the shape we want to search and the definition of a cost function that quantitatively determines whether or not a contour is adjusted to the object. The general outline of the system used is shown in figure 1. As illustrated, for the contour modelling and parametrization the PDM technique [2] has been applied, which lets us restrict all the parameters and precisely define the search space. On the other hand, the objective function proposed is designed to place the contour over areas of the image where edges corresponding to the object are detected. A detailed description of this image analysis stage can be consulted in [5,6].

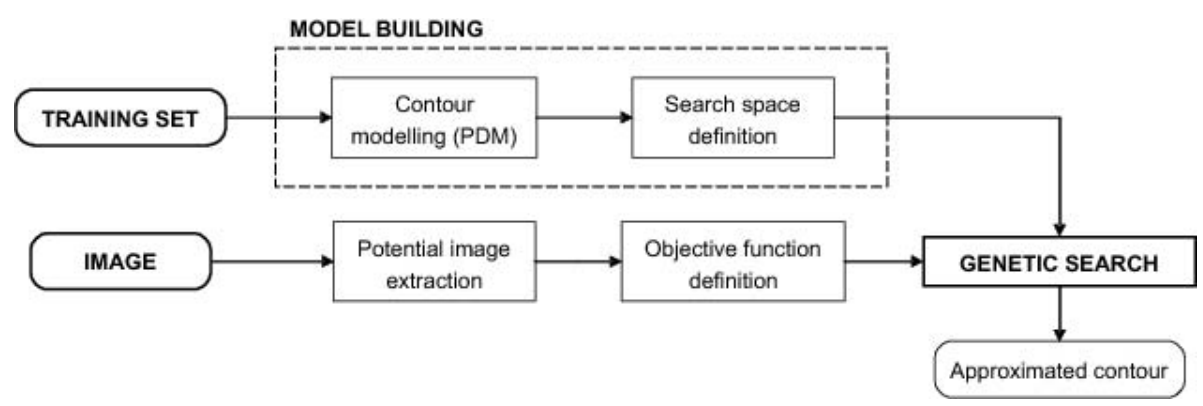

Fig. 1. General outline of the contour location system using genetic algorithms 


\section{Second Stage: Neural Network}

Once the contour of the animal in the image has been located and parameterized, a system is needed to provide an assessment as a function of those parameters. Though our contours are characterized by 14 parameters (the transformation $(s$, $\theta, \boldsymbol{t})$ and 10 shape parameters $b_{i}$, see [6]), we discarded four of them $\left(s, \theta, t_{x}\right.$ and $t_{y}$ ) because they provide information about the position of the animal within the image, but not about the morphology of them. Hence, we use the vector $\boldsymbol{b}$ as input for a multilayer perceptron [1], with one hidden layer, ten neurons at the input and one neuron at the output, which gives us the value for the assessment.

In order to train the network we have a database of images of cows whose morphological assessment made by experts is known. After splitting this set of data into two subsets (training and validation), the training is carried out using the backpropagation algorithm with momentum, and stops when the mean squared error (MSE) obtained over the validation set goes through a minimum. Later, we repeat this process by increasing the network size and we chose the new configuration as optimal if the MSE over the validation set is lower than the previous one.

\section{Experiments and Results}

We made experiments to test both stages of our system. On one hand we wanted to determine the performance of the image analysis stage. On the other hand, we intended to find out about the ability of the system to reproduce the assessments of the experts.

\subsection{First Stage}

We tested our method with 79 images (640x480 pixels size) from our database, to determine the performance of the system with and without fixed points and to compare results. In order to evaluate quantitatively a resulting contour, the mean distance between its points and their proper position has been proposed as a figure of merit. In our case, the distance was estimated by using only seven very significant points of the contour. Those points were precisely located for all the images in the database.

To test the performance of the method we run the GA search without fixed points and with all the possible combinations of one and two fixed points, over the 79 images. In order to define "success", we applied a hard limit of ten pixels to the distance, what we consider enough for the morphological assessment in which these contours are to be used. As a result we obtained that without fixed points the rate of success was $61.3 \%$, but with two fixed points, in the best of the cases (fixing significant points corresponding to the limbs) the rate of success reached $95.7 \%$. However, there was also another case where, fixing 2 points, we obtained only a $58 \%$ of successes. These results indicate that the help of fixed points is very important for the system, but also that the points to be fixed must be well-selected by the operator. 


\subsection{Second Stage: Assessment}

In order to test de morphological assessment system, and considering that we do not have a great amount of data, we have followed a leave-one-out strategy. For each of the 118 images (for which we had the contour extracted) we randomly divided the remaining 117 into two subsets: training and validation. Using this subsets, networks were trained with a number of neurons in the hidden layer between 5 and 40, and we selected the one offering the best results. Finally, the contour from the original image was introduced to the network in order to be assessed, and the result was compared with the assessment provided by the expert, to generate the absolute error.

Table 2. Mean and standard deviation of the absolute value of the error, for the global score and for the individual aspects

\begin{tabular}{clcc}
\hline Trait Description & Mean abs. error & $\sigma$ \\
\hline & Global score & 5.662 & 4.048 \\
\hline 1 & General aspect and figure & 0.666 & 0.469 \\
2 & Development of the body & 0.619 & 0.484 \\
3 & Head & 0.768 & 0.536 \\
4 & Neck, chest, withers and back & 0.832 & 0.493 \\
5 & Thorax and belly & 0.835 & 0.530 \\
6 & Back and loins & 0.717 & 0.528 \\
7 & Hindquarters and tail & 0.881 & 0.745 \\
8 & Thighs and buttocks & 0.809 & 0.659 \\
9 & Extremities & 0.671 & 0.635 \\
10 & Shape and quality of the udder & 1.235 & 0.846 \\
\hline
\end{tabular}

This process has been carried out both with the global score and with the individual scores for each of the aspects. In table 2, the mean and the standard deviation of the absolute value of the error are presented, both for the global score and for the individual aspects. As we can see, we obtain a mean error of 5.662 points in the global score with a relatively high sigma, indicating that there is high variability in the errors of the assessments. We think that the causes for these errors are, mainly, two. First, we have included the limbs in the contours, but the position of the limbs can change significantly from one image to another of the same (or different) animal, causing a great difference between the shape parameters of the contours. Also, though many of the traits to be evaluated can be observed in the image of the lateral position, there are others that can not. The clearest example is the trait 10 (shape and quality of the udder), which obtains the highest error, because the udder is not visible in the contour that we have defined.

Anyway, the results can be considered as quite good and promising, because with a partial input information, we obtain a very low error in many cases. 


\section{Conclusions and Future Research}

In this paper, we have presented a system designed to provide a morphological assessment of a cow based on a lateral image of the animal. The system consist in two parts: one dedicated to the analysis of the image and the extraction of the profile of the animal, and the other designed to provide a score, given the parameterized contour. As we have shown, the first part performs very well with a little human intervention (fixing points), reaching a $95 \%$ of successes. The second stage, based on a neural network, has also given very promising results, taking into account that not all the information required for the morphological assessment can be observed in one view of the animal.

In order to improve the method, two lines are being considered at this moment. First, we are considering to include the contours of the rear and frontal views of the cows as inputs to the neural network, complementing the information contained in the lateral view. Also, we intend to eliminate from the profiles all the parts that are affected by a change in the pose of the animal (for instance, the limbs in the lateral position).

Acknowledgements. This work has been supported in part by the Junta de Extremadura through projects PDT09A045, PDT09A036 and GRU10018.

\section{References}

1. Bishop, C.M., Hinton, G.: Neural Networks for Pattern Recognition. Clarendon Press, Oxford (1995)

2. Cootes, T.F., Taylor, C.J., Cooper, D.H., Graham, J.: Active shape models - their training and application. Comp. Vision and Image Understanding 61(1), 38-59 (1995)

3. Edmonson, A.J., Lean, I.J., Weaver, L.D., Farver, T., Webster, G.: A body condition scoring chart for holstein dairy cows. Journal of Dairy Science 72(1), 68-78 (1989)

4. Goldberg, D.E.: Genetic algorithms in search, optimization and machine learning. Addison-Wesley, Reading (1989)

5. González, H.M., García, C.J., Macías, M., Gallardo, R., Acevedo, M.I.: Application of repeated GA to deformable template matching in cattle images. In: Perales, F.J., Draper, B.A. (eds.) AMDO 2004. LNCS, vol. 3179, pp. 134-145. Springer, Heidelberg (2004)

6. González, H.M., García, C.J., Macías, M., Gallardo, R., Álvarez, F.J.: A method for interactive shape detection in cattle images using genetic algoriths. In: Kropatsch, W.G., Kampel, M., Hanbury, A. (eds.) CAIP 2007. LNCS, vol. 4673, pp. 694-701. Springer, Heidelberg (2007)

7. Goyache, F., Del Coz, J.J., Quevedo, J.R., López, S., et al.: Using artificial intelligence to design and implement a morphological assessment system in beef cattle. Animal Science 73, 49-60 (2001)

8. Haupt, R.L., Haupt, S.E.: Practical Genetic Algorithms 2e. John Wiley, Chichester (2004) 